\title{
BRONKHOPNEUMONIA AS COMORBIDITIES OF CMV INFECTION : STUDY CASE ON 1 YEAR 9 MONTHS OLD BOY
}

\author{
Wulan Widiasmaran ${ }^{1}$, Ayu Anggraini Kusumaningrum ${ }^{2}$, Fitri Amalia ${ }^{3}$, Dimas Tri Anantyo ${ }^{4 *}$ \\ ${ }^{1}$ Undergraduate Program, Faculty of Medicine, Diponegoro University, Semarang, Indonesia \\ ${ }^{2}$ Faculty of Medicine, Diponegoro University, Semarang, Indonesia \\ ${ }^{3}$ Pediatric Specialist Education Program, Faculty of Medicine, Diponegoro University, Semarang, Indonesia \\ ${ }^{4}$ Department of Pediatrics, Faculty of Medicine, Diponegoro University, Semarang, Indonesia \\ *Corresponding author, E-mail : dimastrianantyo@lecturer.undip.ac.id
}

\begin{abstract}
Background. Pneumonia is an acute lung parenchyma inflammation, which includes the alveoli and tissues. According to RISKESDAS 2018, pneumonia prevalence of children under five years old in Indonesia has increased from $1.6 \%$ in 2013 to $2 \%$ in 2018. Cytomegalovirus infection (CMV) is the most common congenital infection and causes high morbidity in newborns. The purpose of this case is to report a case in Semarang and conduct a discussion of pneumonia cases and a history of CMV infection so that proper treatment can immediately start. Case Summary. Patient of a boy aged one year nine months that come to the ER with a chief complaint of fever and cough about five days before entering the hospital. Anamnesis carried out on February 1st, 2020, at Government Hospital in Semarang. The patient was being hospitalized then went sputum culture examination which resulted in the presence of Klebsiella pneumonia, then treated with cefoperazone sulbactam. The patient had a history of cytomegalovirus infection but not optimally treated because of the high avidity value. Conclusion. Patients have diagnosed with bronchopneumonia et causa Klebsiella pneumonia and received therapy in the form of ampicillin and gentamicin for five days, then replaced with cefoperazone sulbactam. The therapy improved the patient's condition.
\end{abstract}

Keywords: Pneumonia, Klebsiella pneumonia, cytomegalovirus infection

\section{INTRODUCTION}

Pneumonia is an inflammation that occurs in the lung parenchyma, which includes the alveoli and surrounding tissue. Pneumonia is still the most significant cause of infant mortality in the world. Pneumonia causes death in 808,649 children < five years old in 2017 . $^{2}$ Based on data from the Riskesdas 2018 shows that the prevalence of pneumonia from $1.6 \%$ in 2013 to $2 \%$ of the population of toddlers in Indonesia in $2018 .{ }^{1}$

Pneumonia is a disease characterized by symptoms of cough, or rapid breathing, and chest wall retractions. Pneumonia can be transmitted through the air, with a source of transmission that is pneumonia sufferers spread microbes in the form of droplets into the air when coughing or sneezing. Then, the microbes that can cause pneumonia disease into the respiratory tract by inhalation or by direct droplet splashes released by the patient when coughing, sneezing and talking inhaled by people around. ${ }^{2}$

Cytomegalovirus Infections (CMV) is a congenital infection that is still prevalent in developing countries. CMV infection also causes high morbidity in newborns. Despite the high CMV infection, few pregnant women aware of the risk of CMV infection that could reach the fetus. ${ }^{3} \mathrm{CMV}$ infection can be obtained before birth or after birth. CMV infection usually occurs asymptomatically in someone with a good immune system, but in someone with an immature immune system (fetus, newborn), depressed (using drugs immunosuppressant) or weak (cancer patients, human immunodeficiency virus, etc.), can cause clinical symptoms that are visible and severe. ${ }^{4}$ Children with CMV infection are more likely to have gastrointestinal malabsorption which can cause malnutrition then leading to low immunity that can cause infection, such as pneumonia. ${ }^{3}$

This paper will describe the case of a patient with bronchopneumonia and history of CMV infection in Government Hospital in Semarang, by describing the findings on history taking, physical examination, supporting examinations, as well as describing the treatment that given at the hospital. Furthermore, this case may inform healthcare delivery, and being a step forward to discuss more about history of CMV 
infection that followed by bronchopneumonia with confirmed Klebsiella pneumoniae.

\section{CASE REPORT}

This case report was evaluated by cross sectional. A boy aged one year 9 came to the Emergency Room of the Government Hospital in Semarang on January 26, 2020, at 09.00 am with the chief complaint of fever since five days ago. Fever accompanied by cough. Three months earlier, patients often experience fluctuating fever, especially at night. The patient's mother does not measure the patient's temperature. Patients have been taken by parents to seek medical treatment from a general practitioner, then given a fever-lowering drug, the fever had dropped after being given a feverlowering medication. Still, after the drug's effects ran out, the child returned fever with no cough, runny nose, nausea, or vomiting. After one week, the child and his parents went to Kendal District Hospital because of a fever that did not heal. In Kendal Hospital, the patient did a laboratory blood examination and found a CMV infection. Then the child is referred to the Government Hospital in Semarang for further management. Patients treated and the virus avidity checked for CMV then obtained a high value (Avidity CMV IgG 0.849), so it does not require CMV treatment. The patient's condition improved, and he was allowed to went home.

Five days ago, the patient's mother complained of the patient's fever returned. The temperature was up and down. The patient's mother measured the patient's temperature and obtained the highest temperature was $39.9^{\circ} \mathrm{C}$. Fever accompanied by coughing and colds, nausea, vomiting, a decrease in appetite. There were no complaints of defecation and urination.

Currently, the child has still had a fever. The child's temperature was $38^{\circ} \mathrm{C}$, the patient only ate a little, the milk ran out through the nasopharyngeal tube, got coughs with mucus purulent.

The patient was born to a 34-year-old mother with G2P1A0 labor spontaneously in the hospital, helped by Obstetrics and Gynecology Specialists. In 37 weeks gestation, the baby was born crying right away with the weight of birth 2600 grams and length of birth $49 \mathrm{~cm}$. During pregnancy, the mother did not have a fever. She had vaccinated during pregnancy and did not consume traditional herbal medicine while pregnant.

Based on KPSP (Kuesioner Pra Skrining Perkembangan) aged 21 months, patients may experience developmental delays because he could not walk or crawl and could only sit.

On the examination of the large fontanel's head has closed and flat, mesocephalic, the conjunctival was not anemic, no nasal lobe breath, no dry mucosa of the mouth, the neck did not get any enlarged lymph nodes. On physical examination of the heart, during the inspection, there were no abnormalities that appeared on the chest. During palpation, there is no thrill of ictus cordis, percussion configuration of the heart within normal limits, auscultation showed regular heartbeat, and no murmurs or gallops. On a physical examination of the lung, obtained symmetrical chest wall inspections, both at static and dynamic, sonor percussion throughout the lung fields, auscultation of vesicular base sounds, and there were rhonchi sounds. On abdominal examination, when an inspection found a flat stomach, normal auscultation of bowel sounds, tympanic percussion, the spleen, and liver were not palpable. Skin turgor quickly returned. The extremities' examination did not reveal cold acral and cyanosis in all four limbs. CRT $\leq 2$ seconds. At the urogenital inspection, the patient has not circumcised, and there was no phimosis.

The general condition is good, pulse $120 \mathrm{x} /$ minute, regular, adequate contents and stress, temperature $38^{\circ} \mathrm{C}$, RR 30x/minute, $99 \%$ $\mathrm{SpO}$ 2. Patients aged one year nine months with weight 9.6 kilograms, height 81 centimeters, upper arm circumference 11 centimeters, and head circumference 46.5 centimeters. WHZ -1.26, WAZ -1.83, HAZ 1.79 , HC -1.13 , so the patient had good nutrition, normal weight, normal stature, and mesocephalic.

Immuno-serological examination (on November 2019) found positive anti-CMV IgG with avidity of 0.849 . X-ray examination of the AP chest supine position (January 27, 2020) showed bronchopneumonia. Sputum culture examination (January 28, 2020) found Klebsiella 
Wulan Widiasmaran, Ayu Anggraini Kusumaningrum,

pneumonia. Routine blood tests (26 January 2020) showed low hemoglobin (10.4 g / dL), low hematocrit (28.0\%), low erythrocytes (3.04 $\mathrm{x} 10^{6} / \mathrm{uL}$ ), normal $\mathrm{MCH}$ (30.3 pg), normal $\operatorname{MCV}(81.6 \mathrm{fL})$, low leukocytes $\left(2.7 \times 10^{3} / \mu \mathrm{L}\right)$, and normal platelets $\left(281 \times 10^{3} / \mu \mathrm{L}\right)$.

\section{DISCUSSION}

Cytomegalovirus infections can occur before birth or after birth. Infants who experience CMV infection during pregnancy can develop into congenital CMV with various morbidity.

CMV infection is so common that almost all adults in developing countries and 50\% to $85 \%$ of adults in the United States have been infected. It transmission can occur horizontally (from one person to another) or vertically (from mother to fetus). CMV is transmitted horizontally through body fluids and requires close contact with body fluids that contaminated with CMV that may found in blood, urine, semen, cervical secretions, saliva, breast milk, and transplanted organs. CMV transmission occurs vertically in the following way ${ }^{5}$; in utero: through the transplacental pathway with CMV viremia in the maternal circulation, intrapartum: fetal exposure to cervical and vaginal secretions containing CMV during labor, postnatal: ingestion of breast milk containing CMV or through blood transfusion that contaminated with CMV.

In this patient, the possibility of transmission is acquired when the patient has been born, likely from the breast milk. At the time of the pregnant women there are no complaints that lead to infection, especially CMV infection.

CMV complications that often occur in infants are developmental disorders in the psychomotor sector. In history, the patient was unable to walk - even by holding or not, and the patient could not walk back five or more steps without losing balance. This, patients may experience developmental delays in the gross motor sector.

Pneumonia is an infection that occurs acutely regarding the alveoli and its surroundings. The air sacs in the lungs called alveoli are filled with pus and fluid to reduce the ability to absorb oxygen. In $2017,15 \%$ of all children under five years' deaths caused by pneumonia. ${ }^{2}$ Pneumonia divided into Community-Acquired Pneumonia (CAP) and Hospital-Acquired Pneumonia (HAP).

Community-Acquired Pneumonia (CAP) defined as pneumonia in patients who do not get hospital stay or long-term inpatient care facilities. ${ }^{6}$ The common bacterium caused by CAP is Streptococcus pneumoniae. And other bacteria that cause CAP include Haemophilus influenzae, Staphylococcus aureus, Moraxella catarrhalis, and other gram-negative bacteria. ${ }^{7}$

According to the criteria from The Centers for Disease Control (CDC-Atlanta), a diagnosis of pneumonia nosocomial are as follows : Onset pneumonia that occurred 48 hours after hospitalization and excluded all an infection whose incubation occurred on admission to the hospital. Diagnosis of nosocomial pneumonia is made on the basis of: Chest X-ray: new or progressive infiltrates Plus 2 among the following criteria: body temperature> $38^{\circ} \mathrm{C}$, purulent mucous, leukocytosis. The criteria for severe nosocomial pneumonia according to ATS : Being treated in an intensive care room, respiratory failure requiring a breath aid or requiring $\mathrm{O}^{2}>35 \%$ for maintains $\mathrm{O}^{2}$ saturation> $90 \%$, progressive radiological changes in the form of multilobar pneumonia or cavities of pulmonary infiltrates, there is evidence of severe sepsis characterized by hypotension and or organ dysfunction: Shock (systolic pressure $<90 \mathrm{mmHg}$ or diastolic $<60$ $\mathrm{mmHg}$ ), requires vasopressors $>4$ hours, urine volume $<20 \mathrm{ml} /$ hour or total urine volume 80 $\mathrm{ml} \mathrm{/} 4$ hours, cute renal failure requiring dialysis.

The pathogens that cause HAP are different from CAP. Nosocomial pneumonia caused by bacteria that are not multidrugresistant (MDR) such as Pseudomonas aeruginosa, Escherichia coli, Klebsiella pneumoniae. Acinetobacter spp and grampositive such as Methicillin-Resistant Staphylococcus aureus (MRSA). ${ }^{8}$

The pathogenesis of pneumonia is related to three factors; immunity of the patient, the microorganisms that attack the patient, and the environment that interact with each other. ${ }^{9}$ The 
presence of bacteria in the lungs is an imbalance condition between the immune system, microbes, and the environment so that microbes can multiply and result in illness.

The diagnosis of pneumonia made by a complete history of the disease, a careful physical examination, and investigation. A definitive diagnosis of pneumonia is made if the chest radiograph contains new or progressive infiltrate plus two or more symptoms such as coughing, changes in phlegm I purulent characteristics, temperature $>38^{\circ} \mathrm{C}$ or history of fever, physical examination reveals signs of consolidation, breath sounds additions such as rhonchi, leukocyte count> 10,000 or $<4500$. $^{10,11}$

The results of the history, physical examination, and supporting examination in this patient concluded that the patient had a cough with purulent mucous accompanied by fever. On physical examination, an additional lung sound was obtained, such as rhonchi, Xray examination showed bronchopneumonia. During routine blood tests on January 28 showed low leukocytes (2700) and Sputum culture examination found Klebsiella pneumonia.

White blood cells are a part of the formation of human blood cells that have a significant role in the immune system to kill germs that enter the human bloodstream. White blood cells or which can also be called leukocytes. ${ }^{12}$ The patient had low leukocytes, which means that the immune system decreased, making it easier for germs to enter the patient's body following the pathogenesis of pneumonia described above. This patient was diagnosed as HAP based on the history and examination that had been done.

Children with CMV infection are more likely to have gastrointestinal malabsorption which can cause malnutrition in the patient. This condition is leading to low immunity that can cause infection such as pneumonia. On the other hand, it can also cause a condition called Global Developmental Delay. ${ }^{3}$

The limitation of this approach is the study was only a case of one patient. It might be not variative enough to discuss about the history of CMV infection history and bronchopneumonia et causa Klebsiella Pneumonia. However, the discussion about this case is quite rare, and it could be a good first step to study about this kind of case, and being a foundation for the further study.

\section{CONCLUSIONS AND SUGGESTION}

The patient of a one year 9 months old baby boy came to the ER with symptoms of fever, up and down for five days. Fever accompanied by cough, but not accompanied by shortness of breath. Coughing has started for five days. Patients were diagnosed with CMV infection in November 2019 but did not get optimal treatment because of high avidity. X-ray examination of the anteroposterior chest supine position revealed bronchopneumonia results. Microbiological analysis of sputum revealed the presence of Klebsiella pneumoniae. Patients were diagnosed with bronchopneumonia and received therapy in the form of ampicillin and gentamicin for five days, then replaced with cefoperazone sulbactam and improved the patient's condition.

\section{THANKYOU NOTE}

The author would like to thank parents of patients who are willing to take their child's history, employees of Government Hospital in Semarang, who took the time to search for the patient's medical record.

\section{REFERENCES}

1. Basic Health Research. 2018. Available from: https://www.who.int/docs/defaultsource/gho-documents/health-equity

2. World Health Organization. Pneumonia. 2019; Available from https://www.who.int/news-room/factsheets/detail/pneumonia

3. Plosa EJ, Esbenshade JC, Fuller MP, Weitkamp J. Cytomegalovirus Infection. Pediatric Rev. 2012; 33 (4): 156-63

4. Chun Soo Kim. Congenital and perinatal cytomegalovirus infection. Korean Paediatrics. 2010; 53 (1): 14-20.

5. Bartlett JG, Dowell SF, Mandell LA, TM File Jr., Musher DM, Fine MJ. Practice guidelines for management of community- 
acquired pneumonia in adults. Infectious Diseases Society of America. Clin Infect Dis. 2000; 31: 347-38

6. Musher DM and Thorner AR, 2014, Community-Acquired Pneumonia, New England Journal of Medicine, 371 (17), 1619-1628.

7. Indonesian Lung Doctors Association. Nosocomial Pneumonia. Guidelines for Management Diagnosis in Indonesia. 2003

8. Dahlan Z. Pneumonia, Internal Medicine. Edition V. Sudoyo AW, editor. Jakarta: Department of Internal Medicine, University of Indonesia; 2009.

9. Mandell LA, Wunderink RG, Anzueto A, et al. Infectious Diseases Society of America / American Thoracic Society consensus guidelines on the management of community-acquired pneumonia in adults. Clin Infect Dis 2007; 44: Suppl. 2, S27 S72.

10. Luttfiya MN, Henley E, Chang L. Diagnosis and treatment of communityacquired pneumonia. American Family Physician. 2010; 73 (3): 442-50.

11. Khasanah MZ, Harjoko A, Candradewi I. Classification of White Blood Cells Based on Color and Shape Characteristics by the K-Nearest Neighbor (K-NN) Method. Indonesian Journal of Electronics and Instrumentations System. October 2016;6(2):151-162 